\title{
Brain abscess from halo pin penetration
}

\author{
Rohit Patel, Bobby K. Desai", T. James Gallagher \\ Department of Emergency Medicine, College of Medicine, University of Florida, Gainesville, USA; \\ *Corresponding Author: bdesai@ufl.edu
}

Received 9 October 2013; revised 7 November 2013; accepted 6 December 2013

Copyright (C) 2013 Rohit Patel et al. This is an open access article distributed under the Creative Commons Attribution License, which permits unrestricted use, distribution, and reproduction in any medium, provided the original work is properly cited. In accordance of the Creative Commons Attribution License all Copyrights (c) 2013 are reserved for SCIRP and the owner of the intellectual property Rohit Patel et al. All Copyright (C) 2013 are guarded by law and by SCIRP as a guardian.

\begin{abstract}
Halo fixation devices are often employed for critically ill or trauma patients with unstable cervical pathologies. These include fractures, spinal decompression and reconstruction procedures. However, the critical care literature has surprisingly little information in regard to associated complications. Perry and Nickel pioneered the initial halo device in 1959 and soon afterward recognized complications associated with its use [1]. They developed a detailed regimen to prevent abnormal pin placement and infections. The details include pin placement in "safe" zones, specific degrees of torque, and techniques to minimize infection risk. Despite a low death rate, a cerebral brain abscess often leads to prolonged neurological morbidity [2]. Seizures and pneumocranium have also been ascribed to intracranial penetration of halo pins $[3,4]$. The following describes a patient with cerebral abscess secondary to halo pin penetration. He then developed several other associated complications during hospitalization.
\end{abstract}

Keywords: Cerebral Abscess; Cranial Pin; Halo Orthosis Device; Inner Table of Skull; Complication

\section{INTRODUCTION}

\subsection{History and Presentation}

A 50-year-old male presented to the University of Florida emergency department (ED) with a history of slurred speech and word finding difficulty. Two months ago, his C2 fracture was stabilized with a halo device. The injury resulted from an ATV accident. At the time of initial discharge, he was neurologically intact. Loose screws had required retightening in clinic five weeks prior to this latest admission. Three weeks prior to arrival in the ED, he had developed swelling of his face and pin sites as well as purulent drainage around the pin sites. This resolved with antibiotic treatment (amoxicillinclavulanate). One day prior to arrival, slurred speech and word finding difficulty developed. The patient stated that he awakened disoriented the night previous, noted slurred speech, and had difficulty with specific words, although he knew what he wanted to say. Two hours later, he felt that the problems had resolved. However, he also indicated that his handwriting looked like "gibberish". After phone consultation, he presented to the neurosurgery clinic. A diagnosis of cerebral infection was considered and the patient was sent to Emergency Department for further workup.

\subsection{Examination}

On examination, the patient was alert, awake, oriented, and hemodynamically stable. Vital signs were within normal limits. No abnormalities or localizing findings were found on neurological examination. Speech was completely intact. There was, however, erythema and a small amount of purulent drainage from the pin sites. He was afebrile and the white blood cell count (WBC) was 7000/cu mm. There were no signs of systemic infection.

\subsection{Initial Treatment and Course}

Head CT showed a new left parietal infarct and adequate healing of the cervical spine fracture (Figure 1). The halo was removed. Continued immobilization was accomplished with a hard cervical collar. MRI \& MRA of the head and neck demonstrated meningitis, left frontal cerebritis, and a left parietal intraparenchymal abscess. The abscess appeared related to skull penetration of the halo screws. Antibioitic treatment was initiated with piperacilin-tazobactam. Surgical intervention was planned (Figures 2(a) and (b)). Initial cultures were not sent. 


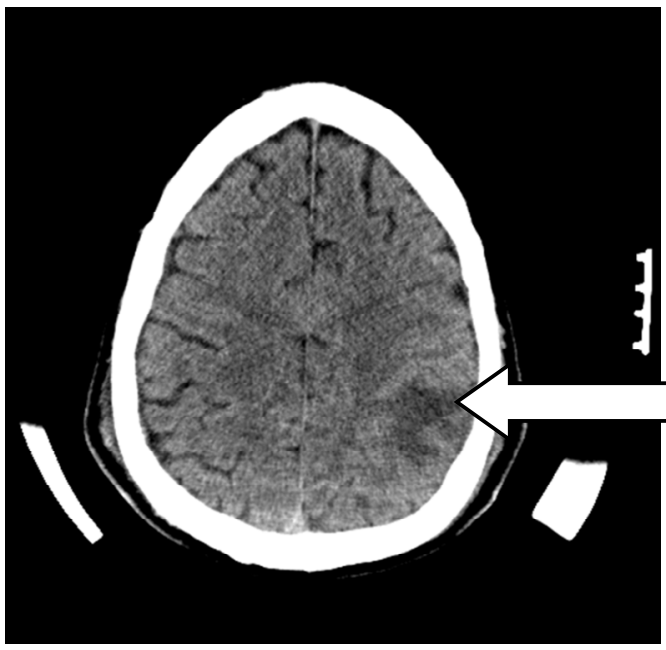

Figure 1. CT brain without contrast. Ill defined area of hypodensity in left parietal lobe with vasogenic edema incompletely visualized due to halo device.

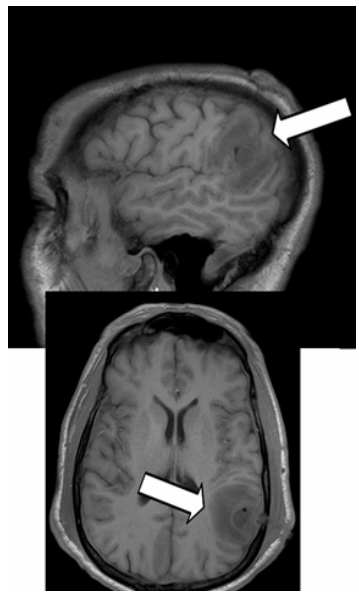

(a)

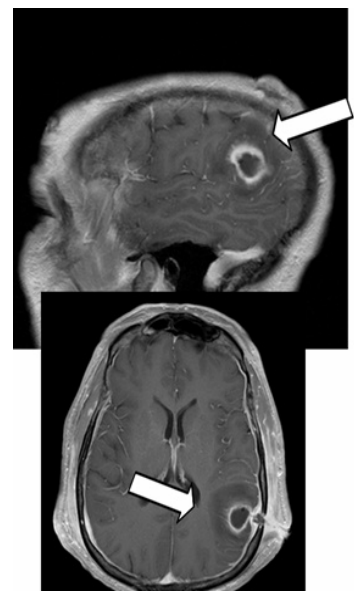

(b)
Figure 2. (a)-(b) MRI T1 pre and post contrast. Intracranial infection with diffuse meningeal thickening and enhancement, $13 \times 16 \times 14 \mathrm{~mm}$ left parietal ring enhancing fluid collection with adjacent vasogenic edema most consistent with abscess.

\subsection{Hospital Course}

Evacuation of the abscess was through a left frontal and parietal craniotomy. Intra-op cultures grew a small amount of MSSA. Two days postop, after being transferred to the floor, the patient experienced an un-witnessed fall. Prior to the event, he had experienced increasing confusion. A nurse went into room to check on the patient and found him on the floor awakes but still confused with his c-collar in place. An emergent CT scan demonstrated a $6.5 \times 4.5 \mathrm{~cm}$ hematoma within the previous left parietal operative bed. He was again returned to the OR for hematoma evacuation (Figure 3). Postop, his neurological status continued to improve. The Infectious Disease service recommended a six-week course of oxacillin.

\section{DISCUSSION}

Cerebral abscess related to a halo screw represents a rare complication and requires early recognition [5]. To date minimal information has appeared in the critical care related literature. Halo devices have many advantages. The halo effectively maintains spinal column alignment. It can be easily applied and has minimal interferences with mandibular function. Furthermore it provides earlier mobilization.

Garfin et al. [6] reviewed 179 cases to identify risks with the external halo device and found that pin loosening occurred in $36 \%$ of patients, pin site infection in $20 \%$, severe pin discomfort in $18 \%$, dysphagia in $2 \%$, and dural penetration in $1 \%$.

A cerebral abscess can occur through improper pin placement with penetration of the (inner table) due topoor hygiene, or loosening or over-torqued cranial pins [7]. Saeed et al. described 16 cases of halo pin associated cerebral abscess [8]. The most common presenting symptom was headache (8 cases), followed by fever (4 cases), nausea/vomiting (3 cases), focal neurological deficits (2 cases), altered mental status (2 cases), and localized pain ( 2 cases). The most common location of the abscess was the parietotemporal area. S. aureus was isolated in 14 of the 16 cases. The remaining cases showed $S$. epidermidis, Peptococcus, or no organisms.

Penetration of the pins through the inner table of the skull can create a direct route into the intracranial cavity. Pins are constructed so that there is a sharp point and a broader body to prevent such occurrence. However, it may still occur particularly in an area of thin bone. "Safe" zones have been recommended such as the posterolateral aspects of the calvaria where the density of bone is greater. This area corresponds to the 4 o'clock and 8 o'clock positions. The 12 o'clock position involves the anterior calvaria or glabella and the 6 o'clock location represents the posterior calvaria or posterior occipital protuberance. Although the scar created by placement

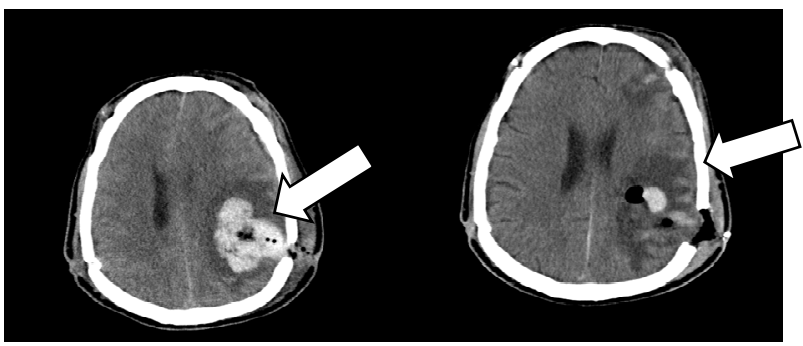

Figure 3. CT without contrast after fall and evacuation (on right). Large left parietal operative site hemorrhage and extra axial blood collection with associated mass effect with herniation and hydrocephalus. 
through the temporalis muscle is within the hairline and more aesthetic, penetration of the pins through the temporalis muscle is painful and may impede the ability to chew without discomfort. The bone underlying this area is also thinner and prone to puncture with repeated pin loosening [9].

Initial pin placement requires appropriate torque to prevent loosening and possible skull penetration. Rizzolo et al. [10] compared 102 patients with a pin torque of either 6 or 8 inch-pounds. Thosepins inserted with 6 inch-lbs torque had fewer complications, including loosening, infection, pin change, or loss of pin. Pande et al. [11] reported a case of transient brain injury from pin penetration into thetemporoparietal region of the brain [11]. This developed 6 hours after the halo was retightened. The patient demonstrated drowsiness, facial asymmetry, and a weak left hand grip. There was no infection and the patient recovered. The pin was replaced.

Poor pin site hygiene can also lead toinfection. Water and mild soap are recommended for cleaning the sites. Agents including povidone-iodine, hydrogen peroxide, and chlorhexidine have shown higher infection rates [12]. Also, excessive cleaning can lead to excess granulation tissue around the pins and subsequent loosening.

Re-tightening of the pins generally occurs at 24 hours and again after 1 week. Also routine follow up should be done at 4 - 6 week intervals.

Antibiotic therapy has been more successful when begun prior to actual abscess formation. Once an abscess develops, surgical evacuation completed with antibiotic therapy becomes the only available option. Some cases have presented as orbital pain, aphasia, seizure, lethargy, disorientation, or psychosis [13,14]. As previously mentioned, even if adequately treated, these symptoms can be associated with serious long term cerebral parenchyma damage. These include interference with the normal propagation of electrical impulses, and the site now becomes a potential seizure focus $[5,6]$.

\section{CONCLUSION}

Patients with halo placement are often in the ICU. Awareness of potential complications from these devices must be part of the clinicians' competence. Those with halo pins and a clinical picture suggestive of infection require close and detailed evaluation. Pin site infection should be promptly treated to prevent pin penetration into the brain and possible deep cranial infection. Early recognition may reduce morbidity.

\section{REFERENCES}

[1] Perry, J. and Nickel, V.L. (1959) Total cervical spine fusion for neck paralysis. The Journal of Bone \& Joint Sur- gery, 41, 37-60.

[2] Ray, A., Iyer, R.V. and King, A.T. (2006) Cerebral abscess as a delayed complication of halo fixation. Acta Neurochirurgica (Wien), 148, 1015-1016. http://dx.doi.org/10.1007/s00701-006-0799-0

[3] Cheong, M.L., Chan, C.Y., Saw, L.B. and Kwan, M.K. (2009) Pneumocranium secondary to halo vest pin penetration through enlarged frontal sinus. European Spine Journal, 18, S269-S271. http://dx.doi.org/10.1007/s00586-009-1004-y

[4] Kingma, R., Peters, J.M. and Coene, L.N. (2006) Intracranial penetration of a halo pin causing an epileptic seizure. The Journal of Bone \& Joint Surgery, 88B, 16541655.

[5] Gelalis, I.D., Christoforou, G., et al. (2009) Brain abscess and generalized seizure caused by halo pin intracranial penetration: Case report and review of literature. European Spine Journal, 18, S172-S175. http://dx.doi.org/10.1007/s00586-008-0759-x

[6] Garfin, S.R., Botte, M.J., Waters, R.L. and Nickel, V.L. (1986) Complications in the use of the halo fixation device. The Journal of Bone \& Joint Surgery, 68A, 320325.

[7] Quinones-Hinojosa, A., Chi, J.H. and Manley, G.T. (2007) Emergent placement of halo orthosis after a traumatic cervical injury leading to a cerebral abscess. Journal of Trauma, 62, E11-E13.

http://dx.doi.org/10.1097/01.ta.0000246938.18656.5d

[8] Saeed, M.U., Dacuycuy, M.A. and Kennedy, D.J. (2007) Halo pin insertion-associated brain abscess: Case report and review of literature. Spine, 32, E271-E274. http://dx.doi.org/10.1097/01.brs.0000259976.46403.8a

[9] Papgelopoulos, P.J., Sapkas, G.S., Kateros, K.T., Papdakis, S.A., et al. (2001) Halo pin intracranial penetration and epidural abscess in a patient with a previous cranioplasty: Case report and review of literature. Spine, 26, E463E467.

http://dx.doi.org/10.1097/00007632-200110010-00030

[10] Rizzolo, S.J., Piazza, M.R., Cotler, J.M., et al. (1993) The effect of torque pressure on halo pin complication rates. Spine, 18, 2163-2166. http://dx.doi.org/10.1097/00007632-199311000-00003

[11] Pande, K., Basu, S. and Webb, J.K. (1998) Transient brain injury from penetration of the halo pin. Spinal Cord, 36, 732-733. http://dx.doi.org/10.1038/sj.sc.3100586

[12] Olson, R.S. (1996) Halo skeletal traction pin site care: Toward developing a standard of care. Rehabilitation Nursing, 21, 243-246.

[13] Victor, D.I., Breshan, M.J. and Keller, R.B. (1973) Brain abscess complicating the use of halo traction. The Journal of Bone \& Joint Surgery, 53A, 635-639.

[14] Rosenblum, D. and Ehrlich, V. (1995) Brain abscess and psychosis as a complication of a halo orthosis. Archives of Physical Medicine and Rehabilitation, 76, 865-867. 